\title{
Gait Analysis after Unilateral Total Hip Replacement Surgery
}

\author{
Sadiq J. Hamandi \\ Biomedical Eng. Dep., Al-Nahrain University \\ Baghdad- IRAQ \\ sadiq_hamandi@yahoo.com marwa.azzawi@yahoo.com \\ Received: 7-Jan.2018 \\ http://doi.org/10.29194/NJES.21040458
}

\author{
Waleed Abdulwahed \\ Orthopedic Dep. \\ University of Baghdad, IRAQ \\ waleedbgh@yahoo.com \\ Accepted: 18-Sep.-2018
}

\begin{abstract}
:
Total hip replacement (THR) is an elective surgical procedure with the primary indication being pain relief. The aim of this study is to analyze gait dynamics for patients after they underwent a unilateral THR surgery and compare it with normal parameters. To investigate the gait dynamics a gait analysis was performed on five patients after they underwent a unilateral THR surgery; only two of them were examined before the surgery. The gait analysis was performed using a digital video camera with two force plates. Kinematics data were obtained from 2D trajectories of seven passive markers using SkillSpector software. MATLAB software has been used for inverse dynamics computation. General gait parameters, Harris Hip Score, joints' angles, forces, moments and powers were obtained during gait cycle. It was found that the average of improvement in Harris Hip Score (for four patients who were examined 1.5, 2.5, 3 and 9 months after surgery) is 61.8 points, which is an indication of pain relief. In the other hand, the general gait parameters were found slightly lower than normal after THR surgery. The average hip reaction force was found to be $2.988 \mathrm{~N} / \mathrm{BW}$, which is within normal range. Also, the average of maximum hip extension and maximum hip flexion angles were found to be 25.69 and -13.524 degree respectively, which both are within normal ranges. Furthermore, hip, knee and ankle moments and powers results showed some abnormality. Therefore as a conclusion, patient satisfaction and functional improvement are not related to general gait parameter. And it is not unusual that gait mechanics improvement would not reach normal after months of recovery. Also, the results of gait dynamics which are from the engineer's perspective are compatible with Harris Hip Score, which is from the physician's perspective, in quantifying surgical results and subsequent recovery progress.
\end{abstract}

Keyword: Total Hip Replacement, Gait cycle, Gait dynamics, Force platform, Harris Hip Score, General Gait Parameter.

\section{Introduction}

The hip joint is a true ball and socket joint. The structure of the hip can be divided into several categories this includes: bones and joints, ligaments, tendon and muscles, nerves and blood vessels (Tortora and Nielsen, 2012). Gait analysis is the systematic study of human motion. It involves measurements of general gait parameters, kinematics and kinetics. General gait parameters consist of stride length, cadence and walking speed. Stride length is the distance covered from initial contact to the next initial contact of the same foot. Cadence is the number of steps in one minute. Walking speed is the distance covered in one second (Kirtley, 2004). Kinematics is the description of the gait in terms of the angles, positions, velocities and accelerations of the body segments and joints. Several techniques are available for measurement of gait kinematics, the most convenient and least costly is using single camera and markers placed on skin that represents action of underlying joints.

It is impossible to be perfectly accurate in digitizing the position of markers. These small inaccuracies lead to digitization noise. The most common filter used is second order Butterworth low pass filter. It was found that the best cutoff frequency is $4.5 \mathrm{~Hz}$ in this study (Whittle, 2007). In the other hand, kinetics is the description of the gait in terms of forces, moments and powers. To represent a whole description of forces and motion the ground reaction forces and moments taken from forceplates is combined with kinematics taken from camera and anthropometric data from tables using inverse dynamics equations to find hip, knee and ankle angles, reaction forces, moments and powers. Joints' moment (torque) shows net balance between agonist and antagonist. Joints' power shows generated or absorbed by muscles (Blajer et al., 2007).

There are long lists of diseases that affect patient's ability to walk but the pathological mechanics of walking falls into four functional categories, which are: pain, deformity (joint contracture, shortening), muscle weakness and sensory loss. Avascular necrosis is condition in which there is loss of blood supply to the bone with subsequent bone necrosis and collapse. Bone is a living tissue so loss of blood supply will end with bone and bone marrow death. If bone death progress, this will lead to bone collapse and loss of sphericity of femoral head and eventually to osteoarthritis (Sen, 2009). Specific gait deviations due to hip disease such as antalgic gait, true 
shortening or functional leg length discrepancy and trendelenburg gait. Antalgic gait is the gait develops to avoid pain while walking. Function leg length discrepancy is when one or both legs are unable to adjust to the appropriate length for a particular phase of gait cycle, in order for natural walking to occur. Stance phase leg should be longer than swing phase leg by planterflex the ankle extended the hip and knee during stance phase and dorsiflex the ankle flex the hip and knee during swing phase. Failure to achieve flexions and extensions of hip, knee and ankle will lead to functional leg discrepancy. Gait modifications to overcome functional leg length discrepancy are circumduction, vaulting hip hiking and steppage. Trendelenburg is bending towards the supporting limb to reduce the forces in the abductor muscles and hip joint during single leg stance (Whittle, 2007).

Total Hip Replacement surgery is done for end stage joint disease. The damaged femoral head is removed and replaced with a metal stem that is placed into the hollow center of the femur. A metal or ceramic ball is placed on the upper part of the stem. This ball replaces the damaged femoral head that was removed. The damaged cartilage surface of the socket (acetabulum) is removed and replaced with a metal socket. A plastic, ceramic, or metal spacer is inserted between the new ball and the socket to allow for a smooth gliding surface (Knahr, 2011).

In the last decades, there has been growing interest in gait analysis spatially in describing and comparing pathological gait pattern (kadaba, 1989). A lot of studies carried out on patients after they underwent a THR surgery to evaluate gait improvements (Madsen et al., 2004) and (Cho et al., 2004). Some of these studies evaluated preoperative gait analysis and compared it with postoperative (Foucher, 2007). Others studied the changes in gait and muscles activation patterns after THR (Carneiro, 2012) or analyzed gait function after THR using different approaches and types of prosthesis(Maurer-Ertl et al., 2015). But none of them compared gait dynamics results, which is the engineer perspective with questionnaire used by physicians such as Harris Hip Score to evaluate surgical results. The aim of this study is to analyze gait dynamics such as joints' angles, reaction force, moments and powers for patients after they underwent a unilateral Total Hip Replacement surgery and compare gait dynamics parameters for unilateral Total Hip Replacement patients after surgery with gait dynamics before THR (if available) and with normal dynamics parameters. Also, compare gait dynamics parameters (engineer perspective) with Harris Hip Score (physician perspective) for unilateral Total Hip Replacement patient.

\section{Patients}

Five male patients from 16 to 51 years are on the list for receiving a THR, volunteered to participate in this study. The inclusion criteria were: ability to walk on a six meters walkway with no aiding cane; no signs of osteoarthritis at other joints of the lower extremities; and no signs of other acute or chronic diseases and conditions that may affect the neuromusculoskeletal system.

All patients participated in post-operative test, only two of them participated in pre-operative test. Four patients received a left THR surgery, only one of them received a right THR surgery .The fifth patient is expecting another THR surgery for the other limb (bilaterally diseased patient).

Patients underwent a THR surgery to reduced pain and improve walking function that was affected by avascular necrosis (AVN) or osteonecrosis. Table 1 lists patients' age, sex, weight in kilogram, height in meters, abnormality and abnormal side.

\section{Methodology}

During each experimental session, the THR patient was asked to put on seven passive markers and his anthropometric parameters were taken from a scale. Then, the THR patient was asked to perform walking trials on a six meter wooden walkway, barefoot and at self selected speed. Three trials were done and the best was chosen.

\subsection{Markers}

Seven passive markers, yellow round stickers, $25 \mathrm{~mm}$ in diameter were located at palpable anatomical landmarks. The marker landmarks approximate the center of rotation of different segments being analyzed. Figure 1 shows the markers placement on patient during gait analysis

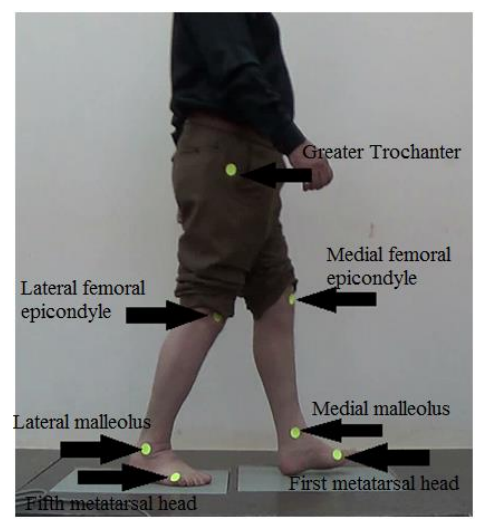

Figure 1: Markers placement on patient's body. 
Table 1: Patients age, six, weight, height, abnormal side and abnormality.

\begin{tabular}{|c|c|c|c|c|c|}
\hline $\begin{array}{c}\text { Patient } \\
\text { number }\end{array}$ & Age & $\begin{array}{c}\text { Weight } \\
(\mathrm{kg})\end{array}$ & $\begin{array}{c}\text { Height } \\
(\mathrm{meter})\end{array}$ & $\begin{array}{c}\text { Abnormal } \\
\text { side }\end{array}$ & $\begin{array}{c}\text { Abnormalit } \\
\mathrm{y}\end{array}$ \\
\hline 1 & 16 & 89 & 1.88 & Left & $\mathrm{AVN}^{*}$ \\
\hline 2 & 29 & 55 & 1.75 & Left & $\begin{array}{c}\mathrm{AVN} * \\
\text { Shortening }\end{array}$ \\
\hline 3 & 50 & 89 & 1.65 & Right & $\begin{array}{c}\mathrm{AVN} * \\
\text { Irregularity } \\
* *\end{array}$ \\
\hline 4 & 51 & 95 & 1.76 & Left & $\begin{array}{c}\text { AVN* } \\
\text { Irregularity } \\
* *\end{array}$ \\
\hline 5 & 36 & 113 & 1.62 & Left & $\mathrm{AVN}^{*}$ \\
\hline
\end{tabular}

*AVN=Avascular necrosis or osteonecrosis

***Irregularity=Irregularity of the head of the femoral.

\subsection{Force Platform}

The force platform is also known as 'force plate' which is used to measure the ground reaction force as subject walk across it. It is important that the subject spontaneously load the force plate as natural event during the course of moving along the walkway, so the force plates mounted on 6 meters flat wooden walkway. Two force plates with different dimensions are available in the laboratory.

Each platform has six components transducer, to measure tiny displacement of the upper surface, in all three axes, when force is applied to it. The electrical output of the platform, six channels, consists of:

1. Three force vector magnitudes (Fx,Fy,Fz)

2. Three moments of force based on the center of the platform (Mx,My,Mz)

To obtain accurate data, it is essential the test foot completely contacts the plate while the other foot remains clear of the plate through the stride. This requires repeating the test several times before the proper foot contact pattern can be attained.

\subsection{Kinematics}

Kinematics system is used in gait analysis to record the angles of the joint and the position and orientation of the body segments, by placing markers on the skin surface in locations that accurately represent the action of the underlying joints. These markers are recorded by a camera and their locations translated into motion data by motion tracking program (SkillSpector).

One camera (50 frames per second) positioned at right angles to the plane of movement $237 \mathrm{~cm}$ away, fitted on a tripod with height of $55 \mathrm{~cm}$ from the ground is used to make a two dimensional measurements. Figure 2 shows gait analysis laboratory dimensions and placement of subjects in the laboratory.

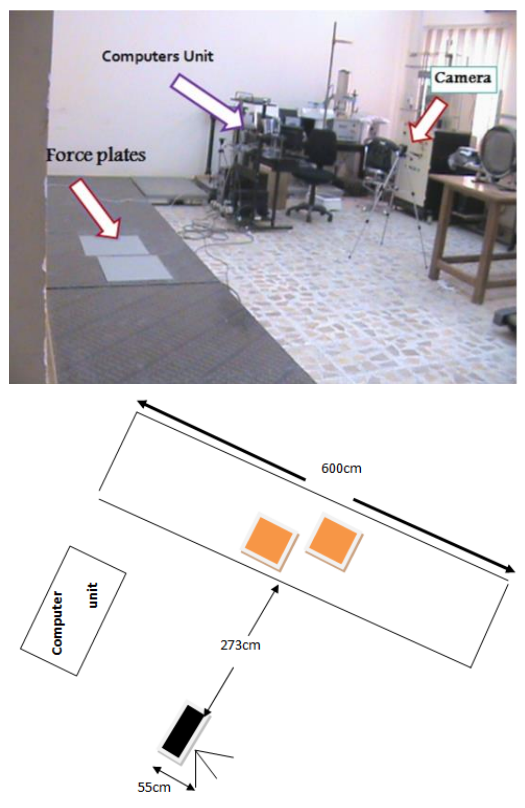

Figure 2: Gait analysis laboratory dimensions and placement of subjects in it.

\subsection{Combined kinetic/kinematic system}

When a kinematic system (camera) is combined with kinetic system (force platform) the capability of the combined system is greater than that of the sum of its component. The reason for this is using inverse dynamic calculations when the relationship is known between limb segment and ground reaction force vector. The limb is treated as a mechanical system in the inverse dynamic tracking program (SkillSpector software) and calculations. This combination is done by special MATLAB software after digitizing the marker movement from camera via the motion analyzer the force plate forces via (Bioanalysis software). Range of angle, power, moment and force in each joint of the lower extremities is the output of the MATLAB program.

\section{Result and discussion}

The following data are results of four patients who were examined 1.5, 2.5, 3 and 9 months after surgery and healthy control.

\subsection{HHS and General gait parameters}

The Harris Hip Score was developed for the assessment of the results of hip surgery. The domains covered in this questionnaire are pain, absence of deformity and range of motion. The pain domain is the most important one which measures pain severity and its effect on activities. Table 2 lists a huge increase in the HHS after THR surgery. The average improvement in HHS 
is 61.8 points. This increased score means a decreased in the pain, which considered as main goal of surgery, although, general gait parameters were slightly lower than normal after the surgery as in Table 3. That is due to walking in closed area and stepping on defined places (force plates); the patients tend to walk slower than normal with shorter steps.
Table 2: HHS results before and after THR

surgery.

\begin{tabular}{|c|c|c|c|}
\hline $\begin{array}{c}\text { Patient } \\
\text { number }\end{array}$ & $\begin{array}{c}\text { Pre- } \\
\text { operative } \\
\text { HHS* }\end{array}$ & $\begin{array}{c}\text { Post- } \\
\text { operative } \\
\text { HHS }\end{array}$ & $\begin{array}{c}\text { Improvement } \\
\text { (points) }\end{array}$ \\
\hline 1 & 27 & 88 & 61 \\
\hline 2 & 58 & 85 & 27 \\
\hline 3 & 29 & 86 & 57 \\
\hline 4 & 2 & 81 & 79 \\
\hline 5 & 0 & 85 & 85 \\
\hline *HHS=Harris Hip Score, the score is out of 100 \\
\hline
\end{tabular}

Table 3: Comparison of general gait parameters between patients pre-and post- operation and normal values.

\begin{tabular}{|c|c|c|c|c|c|c|c|c|c|}
\hline Patient & \multicolumn{3}{|c|}{ Cadence (steps/min) } & \multicolumn{3}{|c|}{ Stride length (m) } & \multicolumn{3}{|c|}{ Velocity $(\mathrm{m} / \mathrm{s})$} \\
\hline & Pre- & Post- & Normal & Pre- & Post- & Normal & Pre- & Post- & Normal \\
\hline $\mathbf{1}$ & 83.33 & 86.95 & $91-135$ & 0.9405 & 1.035 & $1.25-1.85$ & 0.653 & 0.75 & $1.10-1.82$ \\
\hline 2 & 97.23 & 97.24 & $91-135$ & 1.15 & 1.12 & $1.25-1.85$ & 0.931 & 0.908 & $1.10-1.82$ \\
\hline 3 & 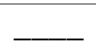 & 65.22 & $82-126$ & & 0.95 & $1.22-1.82$ & & 0.52 & $0.96-1.68$ \\
\hline 4 & & 92.3 & $82-126$ & & 1.02 & $1.22-1.82$ & & 0.774 & $0.96-1.68$ \\
\hline 5 & $\bar{z}$ & 90.2 & $91-135$ & 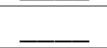 & 0.992 & $1.25-1.85$ & & 0.751 & $1.10-1.82$ \\
\hline
\end{tabular}

\subsection{Hip, knee and ankle angles}

Maximum hip extension angle during terminal stance and pre-swing was lower than normal for all patients after THR according to Figure 3 because even though the artificial hip allows a very wide range of motion it is still less than normal. Besides, muscles around the hip are weak due to being inactive for months or years due to pain. So physical therapy is needed to strengthen the muscles. Lack of physical therapy in our case lowers the extension angle after surgery. The patient who was examined 2.5 months after surgery has higher maximum knee flexion angle and lower maximum ankle planterflexion during initial swing as a compensatory movement to clear the ground.

During initial swing, all patients knee flexion angle were in the ranges of normal values after THR surgery except the patient who was examined 2.5 months after THR according to Figure 3.

Two patients showed higher ankle dorsiflexion during terminal stance and almost all of them showed less ankle planterflexion during initial swing as illustrated in Figure 3.

\subsection{Hip, knee and ankle Reaction Force}

After THR surgery the pain almost vanished which is the main goal of this surgery and the joints reaction force returned to its normal values. Two patients hip, knee and ankle reaction forces had returned to almost normal values. Patient who was examined 9 months after operation the joints reaction forces was higher than normal that is due to other side diseased hip which decreases the force in the affected hip and increases it in the unaffected hip. Patient who was examined 2.5 months after operation the joints reaction forces still lower than normal that is due to abductor muscle weakness. Figure 4 shows normal hip, knee and ankle reaction force and hip, knee and ankle reaction force for patients who were examined $1.5 \mathrm{~m} \mathrm{2.5,3}$ and 9 months after surgery. 

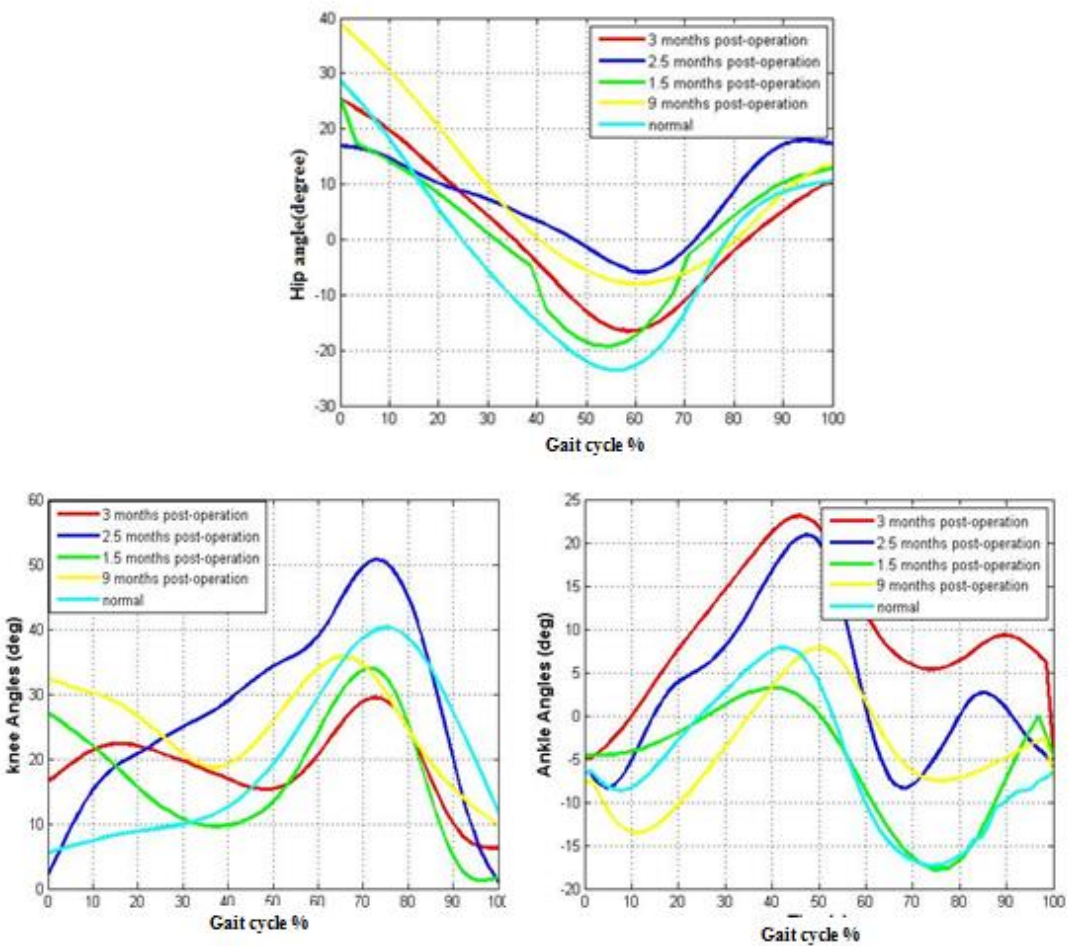

Figure 3: Hip, knee and ankle angle for patients who were examined 1.5, 2.53 and 9 months after surgery and healthy control.
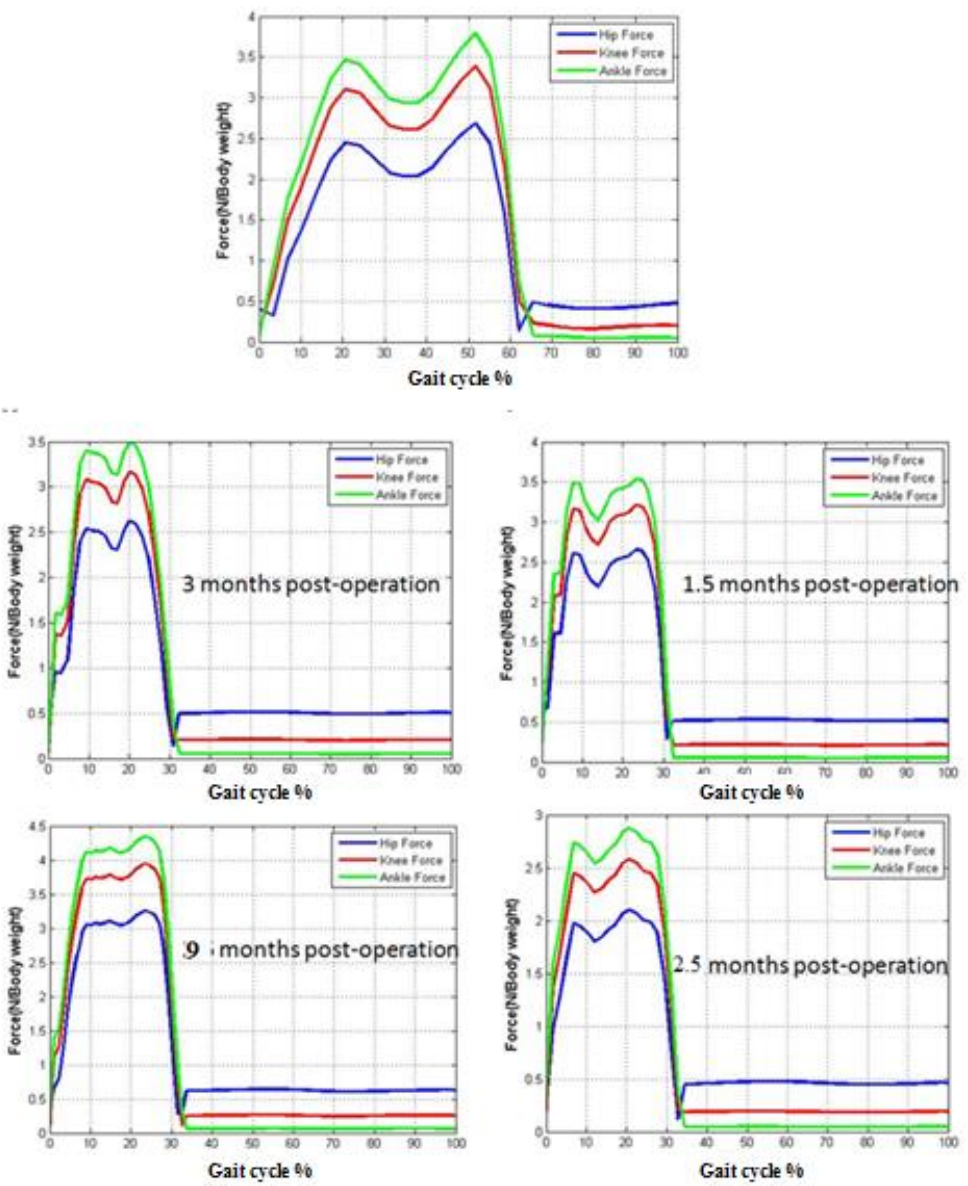

Figure 4: Hip, knee and ankle angle for patients who were examined 1.5, 2.53 and 9 months after surgery and healthy control. 


\subsection{Moments and Power}

Figure 5 shows that maximum ankle planterflexion moment, maximum hip power generation $\mathrm{H} 1$ and maximum knee power absorption $\mathrm{K} 4$ were normal in almost all patients. Maximum hip flexion moment, maximum power absorption $\mathrm{H} 2$ and maximum knee flexion moment during terminal stance were disappeared. Maximum hip extension moment during loading response and initial stance and maximum knee extension moment during mid-stance were higher than normal in all patients. Besides, Maximum knee power absorption $\mathrm{K} 3$ between pre and initial swing and maximum ankle power generation A2 were lower than normal.
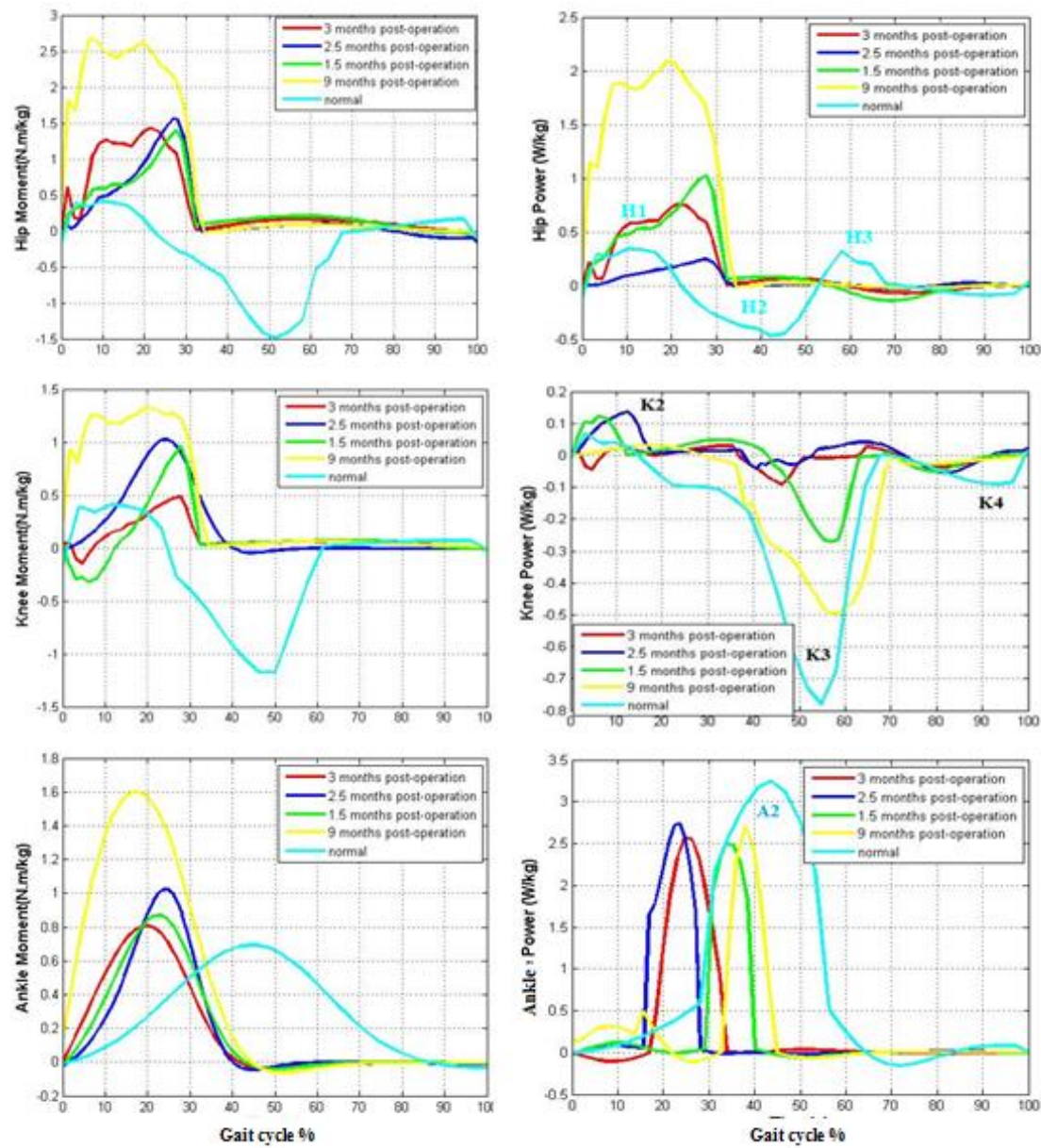

However, although some moments and powers are normal, there are some lower or higher than normal ones which compensate for each other to walk almost normally. So the patients after THR surgery continue walking with some abnormality after months of operation.

Patient number five, who was examined nine months after surgery, shows abnormal joints' moments and powers because of the other antalgic hip. So, bilaterally disabled patients are in a great need for the second THR on the opposite leg if they are to gain optimal function.

The normal values in this study are taken from a study that underwent the same circumstances conducted in the same laboratory (Faihan, 2015).
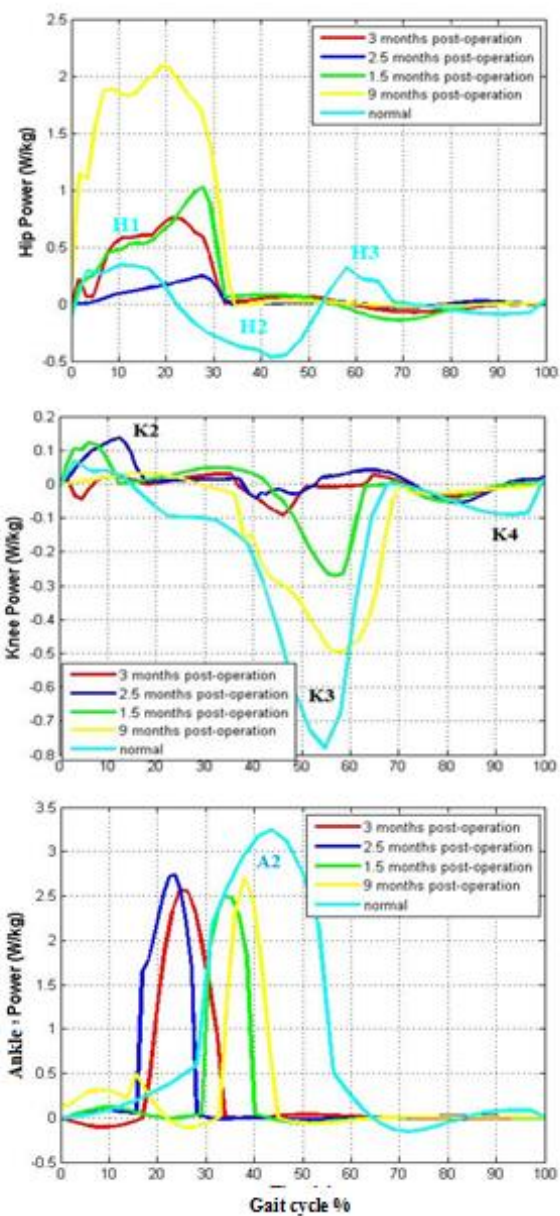

Figure 5 : Hip, knee and ankle moments and powers for patients who were examined 1.5, 2.53 and 9 months after surgery and healthy control. 
Stride length and velocity were slightly decreased after the revision of THR, but the values were still below normal according to Table 2. Cadence did not change but it is in the ranges of normal values. Harris hip score increased from 58 points to 85 points, this patient experienced the lowest improvement among other patients 27 points according to Table 1.

Figure 6 shows a decrease in hip, knee and ankle reaction force after revision of THR surgery due to surgeon advice of minimal weight bearing till union of bone osteotomy (bone cutting).

Figure 7 shows hip joint extended less but flexed more during swing phase after revision surgery. Knee joint flexed more during swing phase and ankle planterflexed less and dorsiflexed more during terminal stance after revision of THR. The excessive flexions and extensions are compensatory movements to clear the ground during stance phase.

After revision of THR surgery hip powers and moments did not change. Knee and maximum ankle planterflexion moment significantly decreased, and generated powers $\mathrm{K} 2$ and $\mathrm{A} 2$ decreased also after revision of THR surgery. Figure 8 and figure 9 show moments and powers for hip, knee and ankle before and after revision of THR surgery.
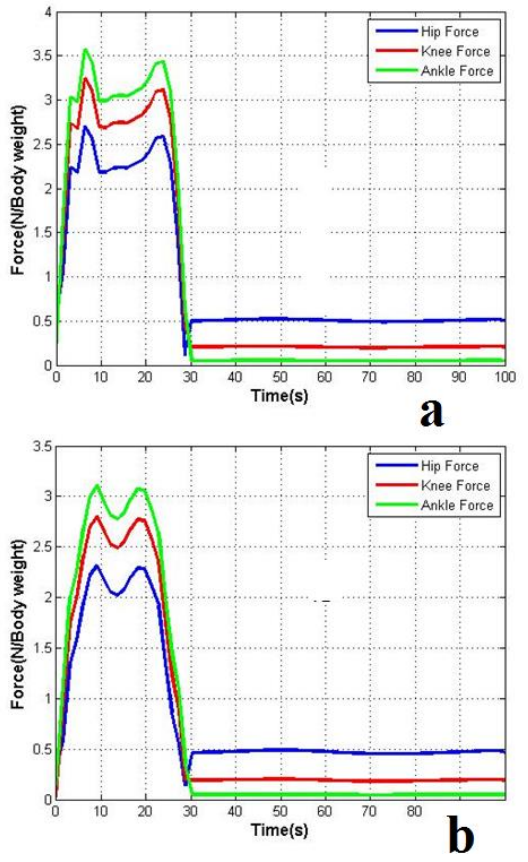

Figure 6 : Hip, knee and ankle joint reaction force a) before THR b) after revision of THR
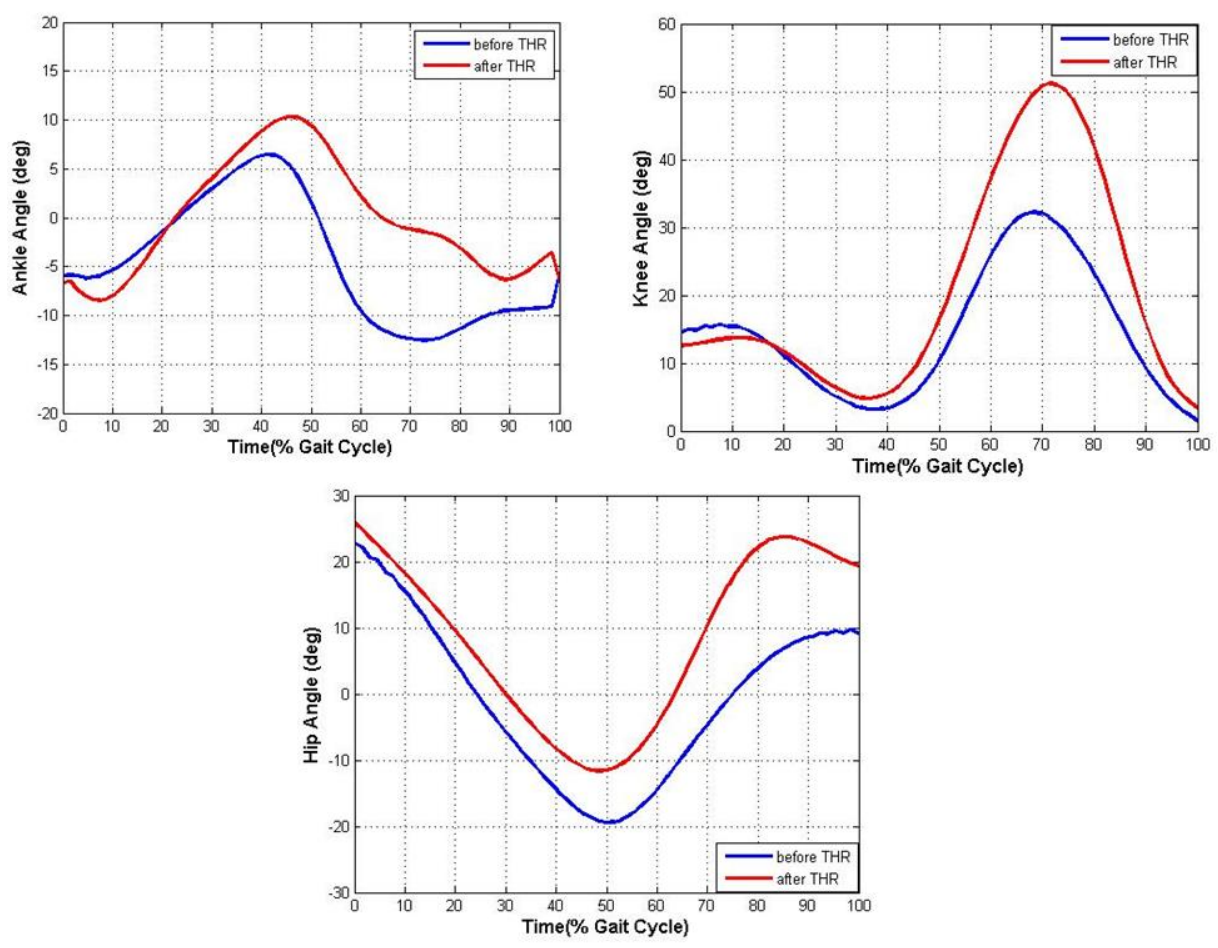

Figure 7 : Hip, knee and ankle joints angle before and after revision of THR surgery. 

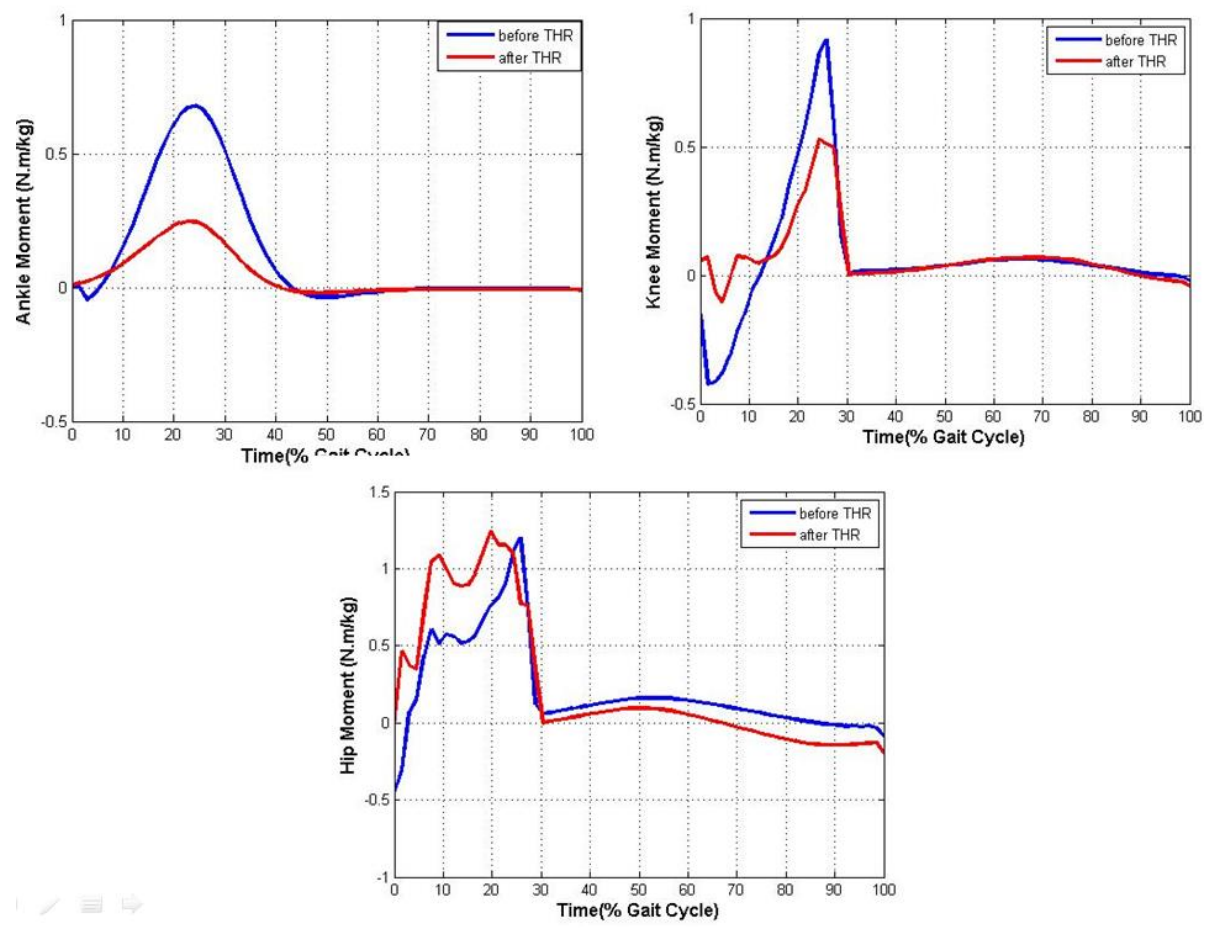

Figure 8: Hip, knee and ankle joints moment before and after revision of THR surgery
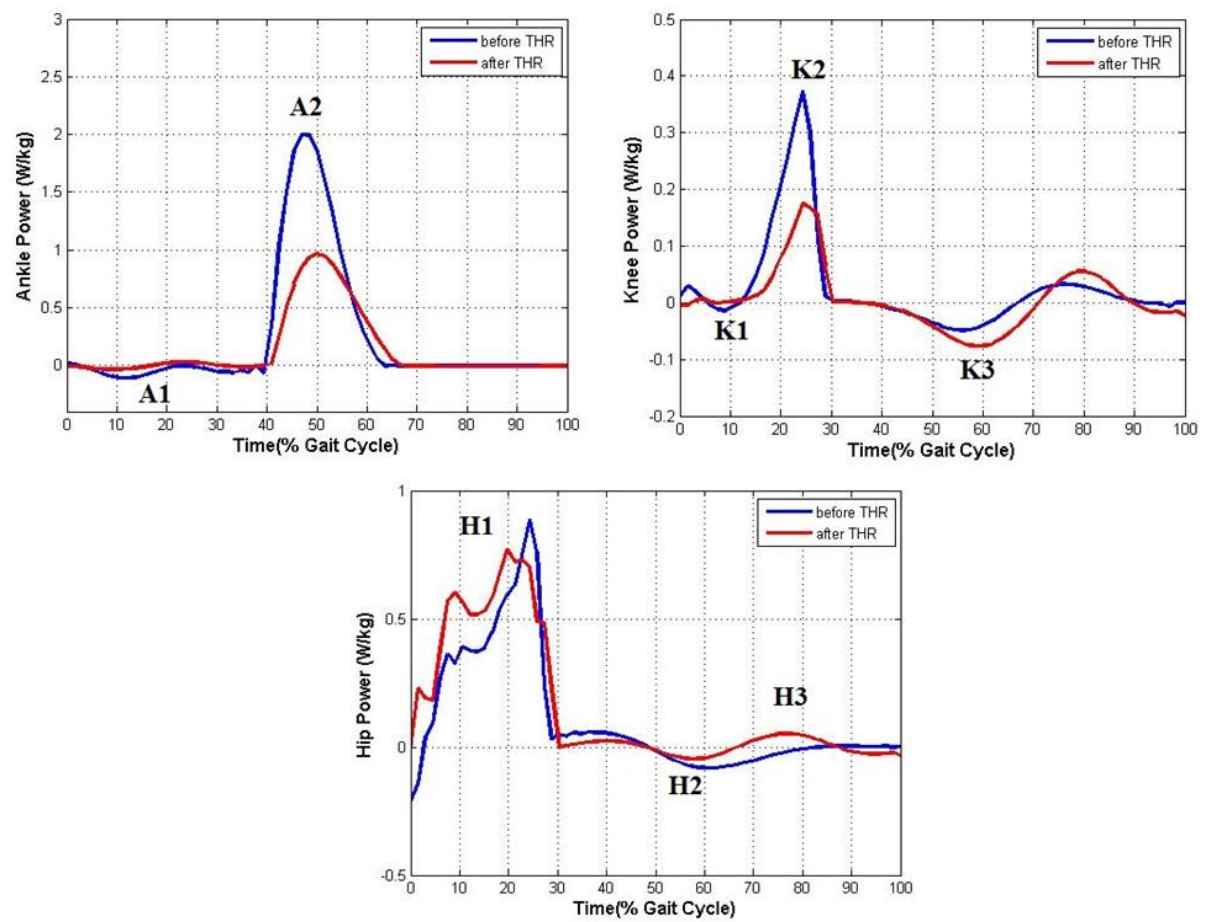

Figure 9: Hip, knee and ankle joints power before and after revision of THR surgery

\section{Conclusions}

In conclusion, it is evident that this study has shown that the use of instruments that have a better sensitivity and specificity than traditional scoring systems such as Harris Hip Score is needed to evaluate the results of THR surgery and enhance the surgeon's ability to assess the overall outcome, allowing a more directed treatment.

The results obtained have indicated that clinical improvement is not parallel to gait parameters improvement. Furthermore, Postoperatively, patients report a reduced amount of pain in the affected hip, and although their gait is 
closer to normal, they often continue to walk with gait abnormalities.

From the outcome of our investigation about special cases it is possible to conclude that gait analysis has confirmed that bilaterally disabled patients are in great need of the second THR on the opposite leg if they want to gain optimal function. And the results of revision THR surgery are unpredictable. Complications are more frequent than the primary surgery.

\section{References}

[1] Blajer, W., Dziewiecki, K., Mazur Z., "Multibody modeling of human body for the inverse dynamics analysis of sagittal plane movements", Multibody System Dynamics, Vol. 18(2) pp. 217-232, 2007.

[2] Cho, M.D., Soo Ho Lee, M.D., Ki Hyung Kim, M.D., and Jong Yoon Yu, M.D., "Gait Analysis before and after Total Hip Arthroplasty in Hip Dysplasia and Osteonecrosis of the Femoral Head", Journal of Korean Orthopedics. 39: 482-8,2004.

[3] Faihan, "Dynamic analysis of human gait cycle", Journal of Al-Nahrain University, 468, 2015.

[4] Foucher, Debra E. Hurwitz, Markus A. Wimmer, "Preoperative gait adaptations persist one year after surgery in clinically well-functioning total hip replacement patients", Journal of Biomechanics, vol. 40, no. 15 pp. 3432-3437, 2007.

[5] Kadaba, H. K. Ramakrishnan, and M. E. Wootten, "Measurement of Lower Extremity
Kinematics During Level Walking”, Journal of OrthopaedicResearch 8383-392, 1990.

[6] Kirtley, "Clinical gait analysis" Theory and practice. ISBN: 044310009 8, 2004.

[7] Knahr, Michael M. Morlock, Nick Bishop, and Gerd Hube "Tribology in Total Hip Arthroplasty", Biomechanics of Hip Arthroplasty, pp.11-24, 2011.

[8] Madsen, Merrill A Ritter, Harold H Morris, John B Meding, Michael E Berend, Phillip M Faris, Vassilios G Vardaxis, "The effect of total hip arthroplasty surgical approach on gait", Final report of the master project, 22 July 2003

[9] Maurer-Ertl, Friesenbichler J, Svehlik M, Holzer L, Sadoghi P, "The Effects of the Surgical Approach on the Postoperative Gait Mechanics Following Total Hip Arthroplasty", Annual Orthopedic Rheumatol 3(4): 1056, 2015.

[10] Sen, "Management of avascular necrosis of femoral head at pre-collapse stage", Indian Journal of Orthopedic; 43(1): 6-16, Jan-Mar 2009.

[11] Susana Moreira Carneiro, "STATISTICAL GAIT ANALYSIS IN PATIENTS AFTER TOTAL HIP ARTHROPLASTY", (Final report of the master project) Instituto Politécnico de Bragança, 2012.

[12] Tortora., Nielsen Mark T., " Principles of Human Anatomy”, 12th edition, Wiley ISBN13: 978-0470567050, 2012.

[13] Whittle," Gait Analysis: An Introduction", 4th edition. Heidi Harrison, ISBN: 9-7807506-8883-3, 2007

\section{التحليل الحركي بعد عملية تبديل مفصل الورك}

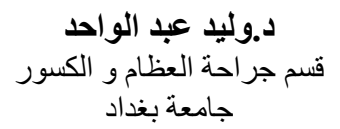

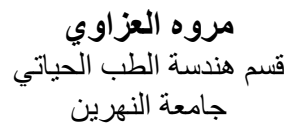

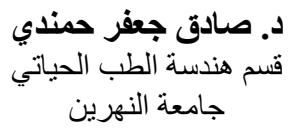

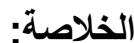

تعد دراسة علم الميكانيك الاحيائي الحركي ماده شامله لتحري اليات العمليه الفسلجيه / العصبيه المعقده التي تسبطر على الأنظمه

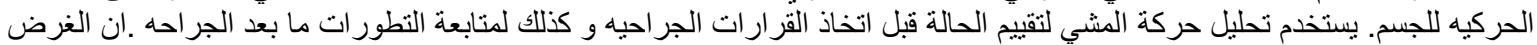

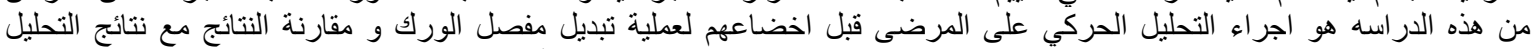

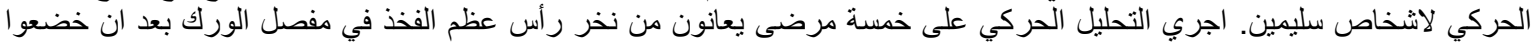

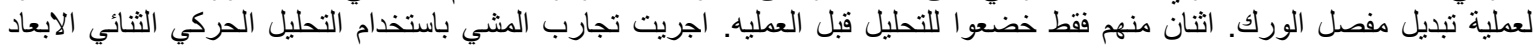

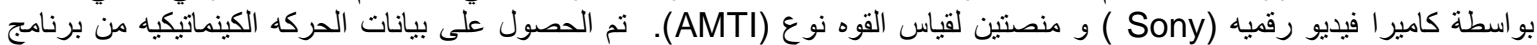
(Skilspector)

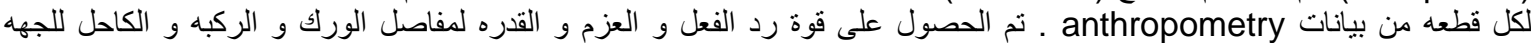

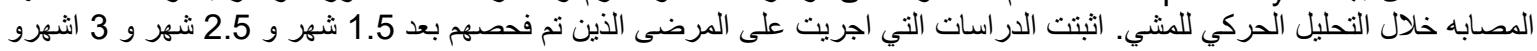

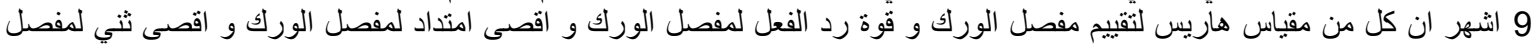

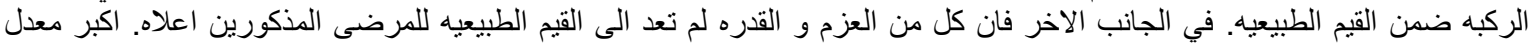

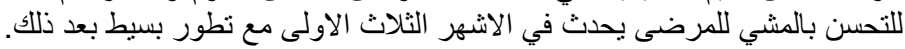

